\title{
Drug shortages in a state-owned academic hospital during the COVID-19 pandemic in Ecuador
}

\section{Jorge Luis Vélez-Páez}

Universidad Central del Ecuador

\section{Diego E. Salazar-Erazo}

Hospital Pablo Arturo Suárez, Ministerio de Salud Pública

Juan P. Castelo-Vigme

Hospital Pablo Arturo Suárez, Ministerio de Salud Pública

Andrea C. Jiménez-Arias

Hospital Pablo Arturo Suárez, Ministerio de Salud Pública

Verónica M. Cevallos-Vique

Hospital Pablo Arturo Suárez, Ministerio de Salud Pública

Lisandro Fernández

Hospital Pablo Arturo Suárez, Ministerio de Salud Pública

Carlos E. Durán

Ghent University

Aquiles R. Henríquez-Trujillo ( $\nabla$ henriquezmd@gmail.com )

Universidad de Las Américas

\section{Research Article}

Keywords: COVID-19, medicine shortages, pharmaceutical procurement, drugs pricing, Ecuador

Posted Date: March 2nd, 2021

DOI: https://doi.org/10.21203/rs.3.rs-289532/v1

License: (c) (i) This work is licensed under a Creative Commons Attribution 4.0 International License.

Read Full License 


\section{Abstract \\ Background}

During a pandemic, medical supplies are at increased risk of being depleted rapidly. The COVID-19 sanitary emergency has generated global shortages in critical supplies, like personal protective equipment and medicines. This study aimed to describe the pharmaceutical procurement challenges to overcome medicine shortages in a state-owned academic hospital during the COVID-19 pandemic in Ecuador.

\section{Methods}

We conducted a cross-sectional study using secondary administrative data of documented public tenders for medicines and drug prescriptions for the critical care unit at Hospital Pablo Arturo Suárez in Quito, Ecuador. The occurrence of shortages, their frequency, and the procurement mechanisms used to overcome them were documented by the pharmacy service between January 1st and July 31th, 2020. The units purchased and unitary price of pharmaceuticals procured by the hospital was also documented and compared to data from 2019.

\section{Results}

From 38 medicines analyzed from 12 ATC groups, there were 84 shortages during the sanitary emergency period covered by the study, and 25 medicines had one or more shortages. Norepinephrine and midazolam shortages were most frequent. Retail or emergency direct purchase and loan-stock from other public hospitals were the main procurement methods to overcome the shortages. The purchase mechanism directly influenced the price. Drugs purchased in small tenders via retail or emergency direct purchase had the most significant price increase (up to 819\%), while medicines acquired via the electronic catalog managed by the public procurement service experienced no price changes.

\section{Conclusion}

Monitoring and responding to drug shortages during a pandemic can be challenging, and governments must adapt quickly, providing specific guidance on how to deal with shortages.

\section{Introduction}

COVID-19, the infectious disease caused by the SARS-CoV-2 virus, was declared a pandemic by the World Health Organization (WHO) on March 11th, 2020.[1] The spectrum of presentation of SARS-CoV-2 infections is highly variable, ranging from asymptomatic infections to severely affected patients with respiratory failure, multiorgan dysfunction requiring critical care, and death.[2, 3] Medical supplies to 
guarantee the standards of care of patients admitted to hospital care during the COVID-19 pandemic had become an increasing challenge worldwide.

Given current global shortages in critical supplies, e.g. personal protective equipment and medicines, pharmacy services' management requires careful coordination between the attending clinicians and the pharmacists to avoid shortages. The aim of this study was to describe the challenges in the pharmaceutical procurement process to overcome medicine shortages in the critical care unit of a stateowned academic hospital during the COVID-19 pandemic in Ecuador.

\section{Methods}

We conducted a cross-sectional study using secondary administrative data of documented public tenders for medicines and drug prescriptions for the critical care unit at Hospital Pablo Arturo Suárez (HPAS), in Quito, Ecuador. The hospital is a state-owned academic hospital managed by the Ministry of Public Health since 1974 and covers approximately $5 \%$ of the 3 million inhabitants of Quito and its surroundings. HPAS has 241 beds, 12 surgical and 20 clinical specialties, including critical care. During the year 2019, there were 13,355 admissions, and 149,372 ambulatory consultations. In the same year, pharmacy service dispensed 312,701 prescriptions, with a total spending of 1.4 million dollars on medicines..[4] On March 11th, 2020, the Ministry of Public Health declared a state of sanitary emergency, HPAS was designated as a COVID-19 hospital, and its critical care unit began to specialize exclusively in managing severe cases of COVID-19.

The presence of drug shortages defined as any shortcoming in the supply of a medicinal product that affects the patient's ability to access the required treatment in due time,[5] and registered as an "undelivered order" in the pharmacy registry, its frequency, and the procurement mechanisms used to overcome the shortage were documented by the pharmacy service personnel between January 1 st and July 31th, 2020. The number of units purchased and the unitary price of pharmaceuticals procured by the hospital were also documented. Drugs were classified according to their ATC code, and medicines consumption and prices during the pandemic were compared to historical records for 2019. All information used for the study was anonymized before the data analysis.

\section{Results}

In total, 38 drug formulations belonging to 12 ATC groups were included in the analysis. From these, 32 formulations presented problems with supply, seven achieve levels below critical stock, and 25 formulations run out of stock at least once during the study period. Only 6 formulations did not experience any shortage (acetylcysteine, amikacin, ampicillin, clarithromycin, risperidone, and salbutamol). There were a total of 84 shortages during the period of sanitary emergency covered by the study, meaning that orders from the critical care unit were not delivered because the stock of the medicine run out, registering an undelivered order. The medicines with more shortages were norepinephrine $(n=9)$ and midazolam $(n=9)$, followed by fentanyl $(n=8)$, rocuronium bromide $(n=6)$, and enoxaparin $(n=5)$. 
The preferred procurement methods to overcome the shortages were purchase via direct tender and stock-loan from other public hospitals. Table 1 presents a detailed description of drug shortages and the procurement methods used to overcome the shortage. 
Table 1

Medicines shortages during the COVID-19 sanitary emergency between march and july 2020, and procurement processes to overcome the shortage.

\begin{tabular}{|c|c|c|c|c|c|c|}
\hline \multirow[t]{2}{*}{ ATC group } & \multirow[t]{2}{*}{$\begin{array}{l}\text { ATC code medicine } \\
\text { and formulation }\end{array}$} & \multirow{2}{*}{$\begin{array}{l}\text { Quantity } \\
\text { below } \\
\text { critical } \\
\text { stock }\end{array}$} & \multirow{2}{*}{$\begin{array}{l}\text { Number } \\
\text { of times } \\
\text { medicine } \\
\text { stock run } \\
\text { out }\end{array}$} & \multicolumn{3}{|c|}{$\begin{array}{l}\text { Type of procurement process } \\
\text { used to overcome shortage }\end{array}$} \\
\hline & & & & Purchase & $\begin{array}{l}\text { Loan } \\
\text { stock }\end{array}$ & Donations \\
\hline \multirow[t]{4}{*}{$\begin{array}{l}\text { B01 } \\
\text { antithrombotic } \\
\text { agents }\end{array}$} & $\begin{array}{l}\text { B01AB05 } \\
\text { Enoxaparin } 40 \mathrm{mg} \\
\text { parenteral solution }\end{array}$ & Yes & 5 & 0 & $\bullet$ & \\
\hline & $\begin{array}{l}\text { B01AB05 } \\
\text { Enoxaparin } 60 \mathrm{mg} \\
\text { parenteral solution }\end{array}$ & Yes & 5 & 0 & 0 & \\
\hline & $\begin{array}{l}\text { B01AB05 } \\
\text { Enoxaparin } 80 \mathrm{mg} \\
\text { parenteral solution }\end{array}$ & Yes & 1 & 0 & & \\
\hline & $\begin{array}{l}\text { B01 AB01 } \\
\text { Nonfractionated } \\
\text { heparin } 5000 \\
\text { Ul/mL parenteral } \\
\text { solution }\end{array}$ & Yes & 3 & 0 & 0 & \\
\hline \multirow[t]{2}{*}{$\begin{array}{l}\text { C01 cardiac } \\
\text { therapy }\end{array}$} & $\begin{array}{l}\text { C01CA24 } \\
\text { Epinephrine } 1 \\
\mathrm{mg} / \mathrm{mL} \text { parenteral } \\
\text { solution }\end{array}$ & Yes & 1 & 0 & & 0 \\
\hline & $\begin{array}{l}\text { C01CA03 } \\
\text { Norepinephrine } 1 \\
\text { mg/mL parenteral } \\
\text { solution }\end{array}$ & Yes & 9 & 0 & & \\
\hline C03 diuretics & $\begin{array}{l}\text { C03CA01 } \\
\text { Furosemide } 10 \\
\mathrm{mg} / \mathrm{mL} \text { parenteral } \\
\text { solution }\end{array}$ & Yes & 4 & & 0 & \\
\hline \multirow[t]{3}{*}{$\begin{array}{l}\mathrm{HO} 2 \\
\text { corticosteroids for } \\
\text { systemic use }\end{array}$} & $\begin{array}{l}\mathrm{H} 02 \mathrm{AB} 02 \\
\text { Dexamethasone } 4 \\
\mathrm{mg} / \mathrm{mL} \text { parenteral } \\
\text { solution }\end{array}$ & Yes & - & & 0 & \\
\hline & $\begin{array}{l}\text { H02AB04 } \\
\text { Methylprednisolone } \\
125 \text { mg parenteral } \\
\text { solid }\end{array}$ & Yes & 3 & 0 & 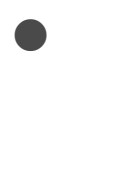 & \\
\hline & $\begin{array}{l}\text { H02AB04 } \\
\text { Methylprednisolone } \\
500 \text { mg parenteral } \\
\text { solid }\end{array}$ & Yes & 2 & & - & \\
\hline
\end{tabular}




\begin{tabular}{|c|c|c|c|c|c|c|}
\hline \multirow[t]{2}{*}{ ATC group } & \multirow[t]{2}{*}{$\begin{array}{l}\text { ATC code medicine } \\
\text { and formulation }\end{array}$} & \multirow{2}{*}{$\begin{array}{l}\text { Quantity } \\
\text { below } \\
\text { critical } \\
\text { stock }\end{array}$} & \multirow{2}{*}{$\begin{array}{l}\text { Number } \\
\text { of times } \\
\text { medicine } \\
\text { stock run } \\
\text { out }\end{array}$} & \multicolumn{3}{|c|}{$\begin{array}{l}\text { Type of procurement process } \\
\text { used to overcome shortage }\end{array}$} \\
\hline & & & & Purchase & $\begin{array}{l}\text { Loan } \\
\text { stock }\end{array}$ & Donations \\
\hline \multirow[t]{11}{*}{$\begin{array}{l}\text { J01 antibacterials } \\
\text { for systemic use }\end{array}$} & $\begin{array}{l}\text { J01GB06 Amikacin } \\
250 \mathrm{mg} / \mathrm{mL} \\
\text { parenteral solution }\end{array}$ & No & - & & & \\
\hline & $\begin{array}{l}\text { J01CR01 } \\
\text { Ampicillin } 100 \mathrm{mg} \\
\text { + beta-lactamase } \\
\text { inhibitor } 500 \mathrm{mg} \\
\text { parenteral solid }\end{array}$ & No & - & & & \\
\hline & $\begin{array}{l}\text { J01FA10 } \\
\text { Azithromycin } 500 \\
\text { mg oral solid }\end{array}$ & Yes & - & 0 & 0 & \\
\hline & $\begin{array}{l}\text { J01FA09 } \\
\text { Clarithromycin } 500 \\
\text { mg oral solid }\end{array}$ & Yes & 1 & & 0 & \\
\hline & $\begin{array}{l}\text { J01FA09 } \\
\text { Clarithromycin } 500 \\
\text { mg parenteral solid }\end{array}$ & No & - & & & \\
\hline & $\begin{array}{l}\text { J01XB01 Colistin } \\
100 \mathrm{mg} \text { parenteral } \\
\text { solid }\end{array}$ & Yes & 4 & 0 & & \\
\hline & $\begin{array}{l}\mathrm{J} 01 \mathrm{DH} 02 \\
\text { Meropenem } 1000 \\
\text { mg parenteral solid }\end{array}$ & Yes & 1 & & & 0 \\
\hline & $\begin{array}{l}\text { J01DH02 } \\
\text { Meropenem } 500 \\
\text { mg parenteral solid }\end{array}$ & Yes & - & 0 & & \\
\hline & $\begin{array}{l}\text { J01CR05 } \\
\text { Piperacillin } 4 \mathrm{~g}+ \\
\text { beta-lactamase } \\
\text { inhibitor } 0.5 \mathrm{~g} \\
\text { parenteral solid }\end{array}$ & Yes & 2 & & & 0 \\
\hline & $\begin{array}{l}\text { J01XA01 } \\
\text { Vancomycin } 1000 \\
\text { mg parenteral solid }\end{array}$ & Yes & 1 & & & \\
\hline & $\begin{array}{l}\text { J01XA01 } \\
\text { Vancomycin } 500 \\
\text { mg parenteral solid }\end{array}$ & Yes & 2 & 0 & 0 & \\
\hline
\end{tabular}




\begin{tabular}{|c|c|c|c|c|c|c|}
\hline \multirow[t]{2}{*}{ ATC group } & \multirow[t]{2}{*}{$\begin{array}{l}\text { ATC code medicine } \\
\text { and formulation }\end{array}$} & \multirow{2}{*}{$\begin{array}{l}\text { Quantity } \\
\text { below } \\
\text { critical } \\
\text { stock }\end{array}$} & \multirow{2}{*}{$\begin{array}{l}\text { Number } \\
\text { of times } \\
\text { medicine } \\
\text { stock run } \\
\text { out }\end{array}$} & \multicolumn{3}{|c|}{$\begin{array}{l}\text { Type of procurement process } \\
\text { used to overcome shortage }\end{array}$} \\
\hline & & & & Purchase & $\begin{array}{l}\text { Loan } \\
\text { stock }\end{array}$ & Donations \\
\hline $\begin{array}{l}\text { M03 muscle } \\
\text { relaxants }\end{array}$ & $\begin{array}{l}\text { M03AC09 } \\
\text { Rocuronium } \\
\text { bromide } 100 \\
\mathrm{mg} / \mathrm{mL} \text { parenteral } \\
\text { solution }\end{array}$ & Yes & 6 & 0 & 0 & \\
\hline \multirow[t]{2}{*}{ N01 anesthetics } & $\begin{array}{l}\text { N01AX10 Propofol } \\
10 \mathrm{mg} / \mathrm{mL} \\
\text { parenteral solution }\end{array}$ & Yes & - & & 0 & \\
\hline & $\begin{array}{l}\text { N01AX10 Propofol } \\
20 \mathrm{mg} / \mathrm{mL} \\
\text { parenteral solution }\end{array}$ & Yes & 1 & 0 & 0 & \\
\hline \multirow[t]{4}{*}{ N02 analgesics } & $\begin{array}{l}\text { N02AB03 Fentanyl } \\
0.05 \mathrm{mg} / \mathrm{mL} \\
\text { parenteral solution }\end{array}$ & Yes & 8 & 0 & 0 & \\
\hline & $\begin{array}{l}\text { N02AA01 } \\
\text { Morphine } 10 \\
\text { mg/mL parenteral } \\
\text { solution }\end{array}$ & Yes & 2 & & 0 & \\
\hline & $\begin{array}{l}\text { N02AA01 } \\
\text { Morphine } 20 \\
\text { mg/mL parenteral } \\
\text { solution }\end{array}$ & Yes & 3 & 0 & 0 & \\
\hline & $\begin{array}{l}\text { N02BE01 } \\
\text { Paracetamol } 10 \\
\text { mg/mL parenteral } \\
\text { solution }\end{array}$ & Yes & - & 0 & & \\
\hline \multirow[t]{4}{*}{$\begin{array}{l}\text { N05 } \\
\text { psycholeptics }\end{array}$} & $\begin{array}{l}\text { N05AD01 } \\
\text { Haloperidol } 5 \\
\text { mg/mL parenteral } \\
\text { solution }\end{array}$ & Yes & 4 & 0 & 0 & \\
\hline & $\begin{array}{l}\text { N05CD08 } \\
\text { Midazolam } 15 \mathrm{mg} / \\
3 \mathrm{~mL} \text { parenteral } \\
\text { solution }\end{array}$ & Yes & 3 & & 0 & \\
\hline & $\begin{array}{l}\text { N05CD08 } \\
\text { Midazolam } 5 \\
\mathrm{mg} / \mathrm{mL} \text { parenteral } \\
\text { solution }\end{array}$ & Yes & 9 & 0 & 0 & \\
\hline & $\begin{array}{l}\text { N05AH04 } \\
\text { Quetiapine } 100 \mathrm{mg} \\
\text { oral solid }\end{array}$ & Yes & 2 & & 0 & \\
\hline
\end{tabular}




\begin{tabular}{|c|c|c|c|c|c|c|}
\hline \multirow[t]{2}{*}{ ATC group } & \multirow[t]{2}{*}{$\begin{array}{l}\text { ATC code medicine } \\
\text { and formulation }\end{array}$} & \multirow{2}{*}{$\begin{array}{l}\text { Quantity } \\
\text { below } \\
\text { critical } \\
\text { stock }\end{array}$} & \multirow{2}{*}{$\begin{array}{l}\text { Number } \\
\text { of times } \\
\text { medicine } \\
\text { stock run } \\
\text { out }\end{array}$} & \multicolumn{3}{|c|}{$\begin{array}{l}\text { Type of procurement process } \\
\text { used to overcome shortage }\end{array}$} \\
\hline & & & & Purchase & $\begin{array}{l}\text { Loan } \\
\text { stock }\end{array}$ & Donations \\
\hline & $\begin{array}{l}\text { N05AH04 } \\
\text { Quetiapine } 25 \mathrm{mg} \\
\text { oral solid }\end{array}$ & Yes & - & ○ & 0 & \\
\hline & $\begin{array}{l}\text { N05AX08 } \\
\text { Risperidone } 1 \\
\text { mg/mL oral } \\
\text { solution }\end{array}$ & No & - & & & \\
\hline $\begin{array}{l}\text { P01 } \\
\text { antiprotozoals }\end{array}$ & $\begin{array}{l}\text { P01BA01 } \\
\text { Chloroquine } 250 \\
\text { mg oral solid }\end{array}$ & Yes & - & & 0 & \\
\hline \multirow[t]{2}{*}{$\begin{array}{l}\text { R03 drugs for } \\
\text { obstructive } \\
\text { airway diseases }\end{array}$} & $\begin{array}{l}\text { R03BB01 } \\
\text { Ipratropium } \\
\text { bromide } 0.02 \mathrm{mg} \\
\text { inhalation solution }\end{array}$ & Yes & 4 & 0 & 0 & \\
\hline & $\begin{array}{l}\text { R03AC02 } \\
\text { Salbutamol } 0.1 \mathrm{mg} \\
\text { inhalation solution }\end{array}$ & No & - & & & \\
\hline $\begin{array}{l}\text { V03 other } \\
\text { therapeutic } \\
\text { products }\end{array}$ & $\begin{array}{l}\text { V03AB23 } \\
\text { Acetylcysteine } 100 \\
\mathrm{mg} / \mathrm{mL} \text { parenteral } \\
\text { solution }\end{array}$ & No & - & & & \\
\hline
\end{tabular}

The purchase mechanism used directly influenced the price. Drugs purchased in small tenders via retail or emergency direct purchase experienced a significant price increase (Table 2). That was the case for norepinephrine, which had the highest price increase (819\%), followed by dexamethasone $(460 \%)$, azithromycin (315\%), and enoxaparin (262\%). Most medicines acquired via the electronic catalog managed by the National Public Procurement Service experienced no price changes. 
Table 2

Changes in unit purchase price of a group of medicines most used in critical care unit before and during COVID-19 pandemic in Ecuador.

\begin{tabular}{|c|c|c|c|c|}
\hline $\begin{array}{l}\text { Main purchase } \\
\text { mechanism during } \\
\text { COVID-19 pandemic } \\
\text { shortage }\end{array}$ & $\begin{array}{l}\text { ATC code and medicine } \\
\text { formulation }\end{array}$ & $\begin{array}{l}\text { Unit purchase } \\
\text { price during } \\
\text { year } 2019 \\
\text { (USD) }\end{array}$ & $\begin{array}{l}\text { Unit purchase } \\
\text { price during } \\
\text { COVID-19 } \\
\text { pandemic (USD) }\end{array}$ & $\begin{array}{l}\text { Price } \\
\text { change } \\
(\%)\end{array}$ \\
\hline \multirow[t]{7}{*}{ Retail } & $\begin{array}{l}\text { C03CA01 Furosemide } 10 \\
\mathrm{mg} / \mathrm{mL} \text { parenteral solution }\end{array}$ & 0.05 & 0.05 & - \\
\hline & $\begin{array}{l}\text { C01CA03 Norepinephrine } 1 \\
\mathrm{mg} / \mathrm{mL} \text { parenteral solution }\end{array}$ & 0.74 & 6.80 & $819 \%$ \\
\hline & $\begin{array}{l}\text { H02AB02 Dexamethasone } \\
4 \mathrm{mg} / \mathrm{mL} \text { parenteral } \\
\text { solution }\end{array}$ & 0.05 & 0.28 & $460 \%$ \\
\hline & $\begin{array}{l}\text { B01AB05 Enoxaparin } 60 \\
\text { mg parenteral solution }\end{array}$ & 1.88 & 6.80 & $262 \%$ \\
\hline & $\begin{array}{l}\text { B01AB05 Enoxaparin } 40 \\
\text { mg parenteral solution }\end{array}$ & 1.68 & 3.48 & $107 \%$ \\
\hline & $\begin{array}{l}\text { B01AB01 Nonfractionated } \\
\text { heparin } 5000 \mathrm{Ul} / \mathrm{mL} \\
\text { parenteral solution }\end{array}$ & 3.00 & 3.50 & $17 \%$ \\
\hline & $\begin{array}{l}\text { R03BB01 Ipratropium } \\
\text { bromide } 0.02 \mathrm{mg} \\
\text { inhalation solution }\end{array}$ & 10.35 & 9.12 & $-12 \%$ \\
\hline \multirow[t]{8}{*}{$\begin{array}{l}\text { Emergency direct } \\
\text { purchase }\end{array}$} & $\begin{array}{l}\text { N05AD01 Haloperidol } 5 \\
\mathrm{mg} / \mathrm{mL} \text { parenteral solution }\end{array}$ & 0.17 & 0.17 & - \\
\hline & $\begin{array}{l}\text { N05CD08 Midazolam } 5 \\
\mathrm{mg} / \mathrm{mL} \text { parenteral solution }\end{array}$ & 0.83 & 0.83 & - \\
\hline & $\begin{array}{l}\text { J01FA10 Azithromycin } \\
500 \text { mg oral solid }\end{array}$ & 0.19 & 0.78 & $315 \%$ \\
\hline & $\begin{array}{l}\text { M03AC09 Rocuronium } \\
\text { bromide } 100 \mathrm{mg} / \mathrm{mL} \\
\text { parenteral solution }\end{array}$ & 2.27 & 4.70 & $107 \%$ \\
\hline & $\begin{array}{l}\text { H02AB04 } \\
\text { Methylprednisolone } 125 \\
\text { mg parenteral solid }\end{array}$ & 6.20 & 7.80 & $26 \%$ \\
\hline & $\begin{array}{l}\text { N02AB03 Fentanyl } 0.05 \\
\mathrm{mg} / \mathrm{mL} \text { parenteral solution }\end{array}$ & 1.35 & 1.50 & $11 \%$ \\
\hline & $\begin{array}{l}\text { N02BE01 Paracetamol } 10 \\
\mathrm{mg} / \mathrm{mL} \text { parenteral solution }\end{array}$ & 1.08 & 1.20 & $11 \%$ \\
\hline & $\begin{array}{l}\text { C01CA24 Epinephrine } 1 \\
\mathrm{mg} / \mathrm{mL} \text { parenteral solution }\end{array}$ & 0.31 & 0.22 & $-29 \%$ \\
\hline
\end{tabular}




\begin{tabular}{|c|c|c|c|c|}
\hline $\begin{array}{l}\text { Main purchase } \\
\text { mechanism during } \\
\text { COVID-19 pandemic } \\
\text { shortage }\end{array}$ & $\begin{array}{l}\text { ATC code and medicine } \\
\text { formulation }\end{array}$ & $\begin{array}{l}\text { Unit purchase } \\
\text { price during } \\
\text { year } 2019 \\
\text { (USD) }\end{array}$ & $\begin{array}{l}\text { Unit purchase } \\
\text { price during } \\
\text { COVID-19 } \\
\text { pandemic (USD) }\end{array}$ & $\begin{array}{l}\text { Price } \\
\text { change } \\
(\%)\end{array}$ \\
\hline \multirow[t]{13}{*}{ Electronic catalog } & $\begin{array}{l}\text { B01AB05 Enoxaparin } 80 \\
\text { mg parenteral solution }\end{array}$ & 0.00 & 6.40 & - \\
\hline & $\begin{array}{l}\text { H02AB04 } \\
\text { Methylprednisolone } 500 \\
\text { mg parenteral solid }\end{array}$ & 10.80 & 10.80 & -- \\
\hline & $\begin{array}{l}\text { J01GB06 Amikacin } 250 \\
\mathrm{mg} / \mathrm{mL} \text { parenteral solution }\end{array}$ & 0.36 & 0.36 & -- \\
\hline & $\begin{array}{l}\text { J01CR01 Ampicillin } 100 \\
\text { mg + beta-lactamase } \\
\text { inhibitor } 500 \text { mg parenteral } \\
\text { solid }\end{array}$ & 0.44 & 0.44 & - \\
\hline & $\begin{array}{l}\text { J01DH02 Meropenem } 500 \\
\text { mg parenteral solid }\end{array}$ & 1.40 & 1.40 & -- \\
\hline & $\begin{array}{l}\text { J01XA01 Vancomycin } 500 \\
\text { mg parenteral solid }\end{array}$ & 1.30 & 1.30 & - \\
\hline & $\begin{array}{l}\text { N01AX10 Propofol } 20 \\
\mathrm{mg} / \mathrm{mL} \text { parenteral solution }\end{array}$ & 4.00 & 4.00 & - \\
\hline & $\begin{array}{l}\text { N02AA01 Morphine } 20 \\
\mathrm{mg} / \mathrm{mL} \text { parenteral solution }\end{array}$ & 0.78 & 0.78 & -- \\
\hline & $\begin{array}{l}\text { N05AH04 Quetiapine } 25 \\
\text { mg oral solid }\end{array}$ & 0.04 & 0.04 & - \\
\hline & $\begin{array}{l}\text { N05AX08 Risperidone } 1 \\
\mathrm{mg} / \mathrm{mL} \text { oral solution }\end{array}$ & 5.70 & 5.70 & -- \\
\hline & $\begin{array}{l}\text { P01BA01 Chloroquine } 250 \\
\text { mg oral solid }\end{array}$ & 0.04 & 0.04 & - \\
\hline & $\begin{array}{l}\text { R03AC02 Salbutamol } 0.1 \\
\text { mg inhalation solution }\end{array}$ & 1.05 & 1.05 & - \\
\hline & $\begin{array}{l}\text { V03AB23 Acetylcysteine } \\
100 \mathrm{mg} / \mathrm{mL} \text { parenteral } \\
\text { solution }\end{array}$ & 0.75 & 0.75 & - \\
\hline \multirow[t]{2}{*}{ Reverse auction } & $\begin{array}{l}\text { J01FA09 Clarithromycin } \\
500 \mathrm{mg} \text { parenteral solid }\end{array}$ & 11.00 & 11.00 & -- \\
\hline & $\begin{array}{l}\text { J01XB01 Colistin } 100 \mathrm{mg} \\
\text { parenteral solid }\end{array}$ & 7.70 & 7.50 & $-3 \%$ \\
\hline
\end{tabular}

Overall spending on medicines increased during the COVID-19 sanitary emergency compared to the previous year. The average monthly spending during the year 2019 was US $\$ 21,232.8 /$ month, in the firsts 
two months of 2020 before the emergency declaration was US\$26,603.7/month and rise to US\$ $55,329.3 /$ month between March and July 2020. The total spending on medicines during 2019 was $\$$ $254,794.5$, and between January to July 2020 , US\$ $329,854.3$.

\section{Discussion}

Our findings show the negative impact of medicines shortages over procurement mechanisms and prices in a public hospital in Quito, Ecuador. During the first months of the Covid-19 crisis, the pharmacy service experienced several episodes of shortages of life-saving drugs for ICU patients. The response was to restock the medicines supply by using the quickest procurement mechanism available by law. The use of retail purchasing proven to have the highest impact on medicines prices (up to $819 \%$ increase in particular cases). Notwithstanding, the provision of medicines through the so-called electronic catalog did not produce price changes during periods of high demand.

Classically, the issue of the shortage is approached based on two main categories, either there is a problem on the demand side exceeding supply at any point in the supply chain and ultimately creating a 'stock out' at the patient's point of service delivery, or the supply of medicines identified as essential is insufficient to meet public health and patient needs.[6] Recently, Acosta et al. have proposed a more comprehensive framework of analysis bringing up 4 principal causes of shortages episodes: market-, supply chain-, manufacturing- and political and ethical issues-related causes.[7]

During the COVID-19 pandemic, several phenomena within these categories have coincided. Firstly, market changes due to an unexpected change in clinical practice.[8, 9] Previous experiences have shown that medicines supplies are at increased risk of being depleted rapidly during a pandemic.[10,11] Secondly, the supply chain management has been deeply affected by the strict lock-downs imposed in countries as China, a prominent producer of active ingredients and medicines. Third, global and local political causes. At the beginning of the crisis, several of the leading worldwide producers of essential goods and medicines closed their borders to protect their own demand, already collapsing the global trade system.[12] From the local side, regulations were not adapted to respond to this new global panorama.

To better understand the context of our study, the Ecuadorian healthcare system comprises two sectors: public and private. The public sector includes all healthcare facilities run by the Ministry of Health (MoH) and those from the public social security subsystems for formal workers, police, and the army.[13] Hospital Pablo Arturo Suárez is one of the MoH specialty hospitals in Quito, Ecuador, and was designated as a COVID-19 hospital during the sanitary emergency.

The medicines supply system for public hospitals relies mainly on a centralized procurement process where preapproved manufactures or wholesalers bid downwards. Once a company wins the bid becomes an exclusive supplier during an established period and is also in charge of distribution to the state-owned health facilities.[14] Thus, healthcare facilities can order and pay for the required medicines through a unique national electronic catalog. 
If the drugs that the health service needs are not available in the electronic catalog, or there is a shortage, a direct purchase is authorized within limited budgets. As our results show, the drawbacks of these purchasing processes outside the traditional bulk procurement mechanisms (national electronic catalog and hospital reverse auction) are the inability to negotiate better prices by losing the advantages of largescale purchases.

Shortages of medicinal products are not a recent problem in Ecuador. Among 7 South American countries, Ecuador was the second-highest on reporting shortages of essential drugs,[15] and the Ministry of Health keeps updating a list of drugs already unavailable in the market for several years.[16] Despite antibiotics are among the most affected group by shortages in previous reports from South American countries,[15] in our study, analgesics, psycholeptics, and inotropic medicines were particularly affected by shortages, which could result from the type of patients allocated to COVID-19 critical care units. A better understanding of how different countries managed the shortages during the COVID-19 pandemic represents an opportunity for learning and improving interdisciplinary collaboration between pharmacists, physicians, and healthcare managers.[17-21]

To avoid abusive drug prices, Ecuador implemented a price regulation system with three regimes: a regulated regime for strategic medicines, a controlled regime for non-strategic medicines, and a direct price setting or emergency regime. The price of medicines is under the regulation of an ad-hoc council that sets and reviews medicines' prices. The council establishes ceiling prices for each market segment, and no drug can be commercialized at a retail price above the ceiling price. However, our results show that the ceiling pricing mechanism had no effect in limiting the price escalations of essential medicines during the pandemic, given that even with considerable increases of up to $800 \%$ in the price, many of the products never exceeded the ceiling prices set by health authorities.

Another finding of this study is that loan-stock from other public hospitals was one of the main procurement methods to overcome the shortages. We have also shown that the purchase mechanism directly influenced the price. Drugs purchased in small tenders had the most significant price increase, while medicines acquired via the electronic catalog managed by the public procurement service experienced no price changes. These findings should lead us to think that integrating the supply chain through shared information systems and creating a single public supply center for essential drugs could be a mechanism to be strengthened in the coming years. Countries like Chile report good experiences with the creation of a Central Supply Clearinghouse, named CENABAST, that procures products for the public health sector, increasing transparency of the pharmaceutical procurement process.[17]

In conclusion, while barriers to accessing medicines are not new, the COVID-19 pandemic poses an urgent need to reinforce effective management strategies for pharmaceuticals at all levels of the supply chain. Monitoring and responding to drug shortages during a pandemic can be challenging, and governments must adapt quickly, providing specific guidance on how to deal with shortages.

\section{Declarations}




\section{Financial support}

There was no external funding for this study or its write-up. Universidad de Las Américas provided unrestricted funding for article-processing charges.

\section{Conflicts of interest}

All authors report no conflicts of interest relevant to this article.

\section{Acknowledgments}

The authors wish to acknowledge the pharmacy service and hospital authorities of Hospital Pablo Arturo Suárez, in Quito, Ecuador, for granting permission to conduct the study.

\section{References}

1. World Health Organization. Listings of WHO's response to COVID-19. 2020. https://www.who.int/news/item/29-06-2020-covidtimeline. Accessed 13 Oct 2020.

2. Ortiz-Prado E, Simbaña-Rivera K, Gómez- Barreno L, Rubio-Neira M, Guaman LP, Kyriakidis NC, et al. Clinical, molecular, and epidemiological characterization of the SARS-CoV-2 virus and the Coronavirus Disease 2019 (COVID-19), a comprehensive literature review. Diagn Microbiol Infect Dis. 2020;98:115094.

3. Montenegro F, Unigarro L, Paredes G, Moya T, Romero A, Torres L, et al. Acute respiratory distress syndrome (ARDS) caused by the novel coronavirus disease (COVID-19): a practical comprehensive literature review. Expert Rev Respir Med. 2020;0.

4. Hospital general provincial Pablo Arturo Suárez. Rendición de cuentas 2019. Quito; 2020.

5. Pauwels K, Simoens S, Casteels M, Huys I. Insights into European Drug Shortages: A Survey of Hospital Pharmacists. PLoS One. 2015;10:e0119322.

6. World Health Organization. Addressing the global shortage of medicines and vaccines Report by the Secretariat. 2016; December:3.

7. Acosta A, Vanegas EP, Rovira J, Godman B, Bochenek T. Medicine Shortages: Gaps Between Countries and Global Perspectives. Front Pharmacol. 2019;10.

8. Vaduganathan M, van Meijgaard J, Mehra MR, Joseph J, O'Donnell CJ, Warraich HJ. Prescription Fill Patterns for Commonly Used Drugs During the COVID-19 Pandemic in the United States. JAMA. 2020;323:2524.

9. Ammassari A, Di Filippo A, Trotta MP, Traversa G, Pierantozzi A, Trotta F, et al. Comparison of Demand for Drugs Used for COVID-19 Treatment and Other Drugs During the Early Phase of the COVID-19 Pandemic in Italy. JAMA Netw Open. 2021;4:e2037060. 
10. Maves RC, Jamros CM, Smith AG. Intensive Care Unit Preparedness During Pandemics and Other Biological Threats. Crit Care Clin. 2019;35:609-18.

11. Mazer-Amirshahi M, Goyal M, Umar SA, Fox ER, Zocchi M, Hawley KL, et al. U.S. drug shortages for medications used in adult critical care (2001-2016). J Crit Care. 2017;41:283-8.

12. Barlow P, van Schalkwyk MC, McKee M, Labonté R, Stuckler D. COVID-19 and the collapse of global trade: building an effective public health response. Lancet Planet Heal. 2021;5:e102-7.

13. Lucio R, Villacrés N, Henríquez Trujillo AR. [The health system of Ecuador]. Salud Publica Mex. 2011;53 Suppl 2:s177-87.

14. Durán CE, Lucio R, Rovira J. Pharmaceutical Policy in Ecuador. In: Pharmaceutical Policy in Countries with Developing Healthcare Systems. Cham: Springer International Publishing; 2017. p. 221-36.

15. ISAGS. Situation of Essential Medicines at Risk of Supply Shortage with Emphasis on South American Countries. Rio de Janeiro; 2017.

16. Ministerio de Salud Pública del Ecuador. MEDICAMENTOS DE DIFÍCIL ACCESO QUE REQUIERE LA RED PÚBLICA INTEGRAL DE SALUD - RPIS PARA EL AÑO 2020. Quito; 2020.

17. Kohler J, Montoya J. Using Technology to Fight Corruption in Pharmaceutical Purchasing: Lessons Learned from the Chilean Experience. 2011.

18. Elbeddini A, Hooda N, Yang L. Role of Canadian pharmacists in managing drug shortage concerns amid the COVID-19 pandemic. Can Pharm J (Ott). 2020;153:198-203.

19. Kuo S, Ou H-T, Wang CJ. Managing medication supply chains: Lessons learned from Taiwan during the COVID-19 pandemic and preparedness planning for the future. J Am Pharm Assoc (2003). 2020.

20. Parreiras Martins MA, Fonseca de Medeiros A, Dias Carneiro de Almeida C, Moreira Reis AM. Preparedness of pharmacists to respond to the emergency of the COVID-19 pandemic in Brazil: a comprehensive overview. Drugs Ther Perspect Ration drug Sel use. 2020;:1-8.

21. Palomar-Fernández C, Álvarez-Díaz A. Hospital Pharmacy Service: Facing the logistics of medicines procurement. Farm Hosp. 2020;44:17-20.

\section{Supplementary Files}

This is a list of supplementary files associated with this preprint. Click to download.

- Table1.xlsx

- Table2.xlsx 